\title{
Formação em saúde e os cenários de aprendizagem no curso de psicologia ${ }^{1}$
}

\author{
Health Training and learning scenarios in the course of psychology
}

Cássia Beatriz Batista $^{[a]}$, Daniele Souza Carmona ${ }^{[b]}$, Sara Lopes Fonseca[ ${ }^{[c]}$

${ }^{[a]}$ Universidade Federal de São João Del Rei - MG - Brasil, e-mail: cassiabeatrizb@gmail.com

${ }^{[b]}$ Pontifícia Universidade Católica de Minas Gerais (PUC Minas), Belo Horizonte, MG - Brasil, e-mail: anamnesedani@yahoo.com.br

${ }^{[c]}$ Pontifícia Universidade Católica de Minas Gerais (PUC Minas), Belo Horizonte, MG - Brasil, e-mail: saralopesfonseca@yahoo.com.br

Recebido: 30/12/2011 Received: $12 / 30 / 2011$

Aprovado: 06/11/2012 Approved: $11 / 06 / 2012$

\section{Resumo}

Esta pesquisa se insere no contexto da formação de recursos humanos para a saúde que, na busca de melhor preparação dos profissionais para atuar no modelo do Sistema Único de Saúde, incentiva mudanças nos cursos de graduação de saúde das universidades brasileiras. Visando caracterizar a formação em saúde da PUC Minas, unidade São Gabriel, a luz das diretrizes curriculares e a partir do tripé ensino, pesquisa e extensão, mapeamos os cenários de aprendizagem em saúde existentes entre os anos de 2006 e 2008, período anterior a entrada do curso para o Programa Nacional de Reorientação da Formação Profissional da Saúde (PróSaúde). Para tal mapeamento, utilizou-se a análise documental do projeto político-pedagógico do curso, relatórios e outros documentos sobre estágios supervisionados, pesquisa, extensão e monografia, além da produção dos docentes. Os campos da saúde e da saúde pública, bem como a abordagem interdisciplinar estão presentes na proposta do curso. Observa-se no período estudado a presença diversificada de cenários de aprendizagem e de temáticas em saúde, mesmo que alguns desses estejam pouco integrados ou ainda dispersos na formação e na articulação entre ensino, pesquisa e extensão. A configuração dos cenários de aprendizagem reafirma a proposta de reorientar a formação em saúde defendida pelo programa, principalmente na atuação multiprofissional e na abordagem interdisciplinar, ainda pouco presentes nos cenários de aprendizagem analisados. Explorando fontes documentais, compreendemos uma dada realidade de formação em saúde que ultrapassa as prescrições curriculares, e apresenta o currículo como possibilidade de construção de sujeitos.

Palavras-chave: Educação Superior. Saúde Pública. Currículo. Atuação (Psicologia).

\section{Abstract}

This research is part of the discussion on the formation of health human resources for health, in an attempt to find well-prepared professionals to act in the Brazilian Health System (Unified Health System - SUS), encouraging changes in the undergraduate health courses of Brazilian

1 A pesquisa contou com a colaboração de três bolsistas de iniciação científica e teve financiamento do Fundo de Incentivo à Pesquisa (FIP), da instituição universitária a qual se vinculam as pesquisadoras.

Psicol. Argum. 2014 jul./set., 32(78), 17-25 
universities. In order to characterize the health training of a private university of Minas Gerais (PUC Minas), regarding the national curriculum guidelines and the tripod of university education, we mapped the learning scenarios in health between 2006 and 2008, prior to the entering of the course to the National Program for the Reorientation of Vocational Training of Health (Pró-Saúde). For doing such work, we used documentary analysis of the political and pedagogic project of the course, reports and other documents on supervised internships, research, extension programs and articles, besides the production of the professors. The fields of health and public health, as well as an interdisciplinary approach are present in the course guidelines. It was observed in the studied period the varied presence of learning scenarios and health themes, even if they can be poorly integrated or dispersed in the training process and in the articulation of teaching/ learning, research and extension. The configuration of learning scenarios reaffirms the proposal to reorient the health education defended by the program, especially in multiprofessional actions and in the interdisciplinary approach, still present in some of the analyzed learning scenarios. Exploring documentary sources, it was possible to understand the given reality of health education that goes beyond the curriculum requirements, and presents the curriculum as a possibility for the construction of subjects.

Keywords: Higher Education. Public Health. Curriculum. Acting Out (Psychology).

\section{Introdução}

O Movimento da Reforma Sanitária Brasileira e a constituição do Sistema Único de Saúde (SUS) expressam a necessidade de adequação da formação de profissionais de saúde mais próxima da realidade da saúde da população assistida, passando então a exigir mudanças tanto educativas quanto organizativas, conceituais e ideológicas. Para além de uma educação instrumental, busca-se modificar o projeto político-pedagógico dos cursos, tendo como norte os princípios da equidade, universalidade e integralidade da política de saúde do país.

A necessidade de se ultrapassar uma formação pautada em uma ciência das doenças, em bases puramente biológicas, com práticas centradas na medicalização e no hospital e com os saberes departamentalizados é mobilizadora da elaboração das Diretrizes Curriculares Nacionais (DCN) para os cursos superiores em saúde. Com as DCN (2004), o Ministério da Saúde e o Ministério da Educação sinalizam a direção da reorientação da formação, buscando modificar o perfil dos recursos humanos da saúde.

A perspectiva da pedagogia libertadora marca essa nova proposta educativa que consistiria, conforme Carvalho e Ceccim (2007), em uma formação que incentivasse o educando a participar, construir e se responsabilizar pelo seu processo educativo, colaborando, desse modo, para o desenvolvimento da capacidade crítica, autoanalítica e autogestionária dos futuros profissionais. Além dessa proposta, os autores apontam a articulação entre o ensino e o serviço, e os currículos integrados como formas de alcançar mudanças na formação.

Todavia, mesmo que o SUS tenha assumido um papel ativo de reorientação do processo de formar e de cuidar da saúde individual e coletiva, provocando importantes repercussões nas estratégias e modos de ensinar e aprender, ainda não se estabeleceu como uma forte potência na formação profissional (Ceccim \& Feuerwerker, 2004).

Para fortalecer essa nova perspectiva de formação e reorganizar o sistema de saúde, focar apenas os profissionais da rede não é suficiente. É necessário repensarmos os cursos de graduação em saúde e a aproximação entre os profissionais do serviço e os estudantes (futuros profissionais), incentivando o diálogo entre a universidade e a rede de serviços. Nesse sentido, o Ministério da Saúde tem apontado caminhos para a formação de profissionais, argumentando que a educação permanente seria uma ação estratégica para o desenvolvimento do SUS ao investir na formação dos sujeitos para esse novo modelo de assistência (Brasil, 2004).

Como incentiva os Ministérios da Saúde e da Educação por meio de diversos programas e ações, temos o Programa Nacional de Reorientação da Formação Profissional em Saúde (publicação do primeiro edital em 2005), que objetiva a integração ensino-serviço de saúde. 0 programa Pró-saúde estimula a construção de parcerias e a cooperação entre as universidades e o sistema de saúde e busca 
efetivar mudanças nessa relação com ações de adoção e desenvolvimento de currículos integrados, de metodologias ativas e inovadoras de ensino-aprendizagem e de avaliação; e ainda com diversificação dos cenários de prática e enfoque no trabalho multiprofissional para garantir a integralidade e a continuidade da atenção e produção de conhecimentos relevantes para o SUS (Brasil, 2004).

Para essa mudança curricular, o Pró-Saúde (Brasil, 2009) propõe, ainda, repensar o perfil do profissional desejado, o tipo de conhecimento valorizado e sua importância não só na articulação entre teoria e prática, mas também em uma perspectiva interdisciplinar. E, para avaliar a mudança, aponta três dimensões do currículo a considerar: o currículo formal e expresso; o currículo real, que é praticado na sala de aula; e o currículo oculto, que transmite valores e mensagens ideológicas que formam as subjetividades $-\mathrm{S}$, pp.

O estudo do currículo destaca a relevância desse documento para a formação de profissionais de saúde no ensino superior. Esses profissionais irão compor e (re)construir cotidianamente o modelo de assistência em saúde do país. Com esse enfoque, pautado pelas discussões sobre a formação para o SUS, sobre as DCN dos cursos de saúde e pelo currículo como documento de identidade (Silva, 2003), investigamos o curso de psicologia da Pontifícia Universidade Católica de Minas Gerais (PUC Minas), que desde 2008 compõe o projeto Pró-Saúde II elaborado em parceria com a Secretaria Municipal de Saúde e com demais cursos de saúde dessa universidade.

A formação promovida pelo curso estudado foi caracterizada pelo projeto educacional do curso e pelo mapeamento dos cenários de aprendizagem em saúde já existentes antes da implementação do programa Pró-Saúde. A análise foi demarcada pelo tripé da formação universitária - ensino, pesquisa e extensão -, assinalando a formação em saúde do ensino superior em seu currículo vivido e mostrando aproximações e distanciamentos dessa formação com a reorientação proposta pelo Ministério da Saúde, bem como os desafios na produção de sujeitos para atuação no SUS.

\section{Percurso metodológico}

Na realização desta investigação, partimos de um caso específico do curso de graduação em psicologia em uma universidade privada para retratar alguns aspectos da experiência curricular que compõe a história coletiva de formação em saúde no ensino superior brasileiro (Minayo, 1994; Becker, 1999). Adotamos a análise de documentos sobre o currículo vivido e seus cenários de aprendizagem, o que nos permitiu sistematizar e analisar informações que foram produzidas por certos atores, uma dada instituição e para destinatários determinados (Borrione \& Chaves, 2004). Buscando apreender uma dada realidade ao explorar fontes documentais e localizando os lugares de produção desses enunciados na instituição universitária, utilizamos também, para a análise dos dados, um enfoque temático na saúde pública e nas atividades de pesquisa, de ensino e de extensão desenvolvidas pelo curso, constituindo assim os cenários de aprendizagem em saúde analisados.

A coleta de dados foi realizada em 2010, e o recorte da pesquisa refere-se aos anos de 2006 a 2008, que precedem a implementação do Pró-Saúde no curso, ocorrido em meados de 2008. Desse modo, o estudo também forneceu um diagnóstico situacional da formação em saúde antes do programa, possibilitando, futuramente, uma análise sistemática com estudos avaliativos e comparativos.

$\mathrm{O}$ acesso aos documentos contou com a colaboração da coordenação ampliada do curso, que disponibilizou o projeto político-pedagógico do curso, relatórios e projetos de estágios, pesquisas, monografias e projetos de extensão e de laboratórios de pesquisa. Complementamos essas informações e obtivemos outras, acessando as publicações e os currículos dos docentes na Plataforma Lattes. A busca inicial pelos termos Saúde e Saúde Pública em títulos e resumos orientou o mapeamento dos cenários de aprendizagem que, por sua vez, foram organizados em projetos de estágios, de pesquisa e de extensão, monografias, publicações e eventos científicos.

Sabemos que o conceito de saúde ou saúde como área profissional envolve uma ampla e importante discussão para a formação no ensino superior, principalmente para pensarmos a formação em psicologia de cursos de graduação em saúde. 0 curso de psicologia prevê habilidades e competências que estão além da saúde pública ou mesmo da saúde para alguns. Atuações como seleção, avaliação e capacitação profissionais ou, ainda, em áreas como educação, sistema jurídico, comunidade, políticas

Psicol. Argum. 2014 jul./set., 32(78), 17-25 
de assistência e desenvolvimento sociais, dentre outras, são, por vezes, situadas em outro campo teórico e profissional. Contudo, nos limitaremos neste artigo à utilização dos termos saúde/saúde pública e em sua apropriação nos cenários de aprendizagens na graduação em psicologia.

Dentre os que utilizam o termo Saúde explicitado nos documentos elegemos aqueles que apresentam uma compreensão de saúde em contexto coletivo ou articulado à cultura, à política ou a programas de saúde, excluindo, então, atividades específicas da chamada clínica (individual ou tradicional), como alguns casos de estágios na clínica-escola. Esse seria um ponto a ser futuramente problematizado: saúde, clínica-escola e saúde pública.

Com esses dados coletados, elaboramos uma planilha com filtros que permitiram cruzamentos de dados entre modalidades de cenários, temáticas da saúde, campos de atuação, instituições, professores e ano de realização da atividade.

Os documentos nos forneceram uma aproximação da realidade estudada, retratada pelo discurso oficial registrado nos relatórios e projetos institucionais analisados. Como em toda pesquisa, há contornos que configuram a produção dos dados e, além dessa caracterização colocada, destacamos as limitações no acesso aos documentos e às fontes que, por vezes, não se encontravam arquivados nem sistematizados ou atualizados. Todavia, o conhecimento da equipe de pesquisa sobre o contexto pesquisado devido a sua inserção como docente, discentes e bolsistas de iniciação científica na instituição, trouxe para o estudo agilidade e facilidade de acesso aos documentos internos do curso e a outras fontes, além das possibilidades de compreensão e de análises do caso vivido pelas pesquisadoras.

\section{Resultados: conhecendo o currículo de psicologia}

A condição integral da atenção em saúde foi fragmentada em especialidades e setores do serviço, e para enfrentar os saberes e as práticas compartimentadas, as DCN sinalizam para uma formação capaz de lidar com os diversos contextos de atuação institucionais e sociais e com as outras profissões. Desse modo, propõe uma articulação intersetorial e interdisciplinar nos currículos, que proporcione uma formação com conhecimentos, competências e habilidades profissionais específicos para atuar de forma integral na saúde.
A dimensão da integralidade no SUS é defendida por diversos autores como o eixo para reorientação da formação e reorganização da assistência em rede (Ceccim \& Feuerwerker, 2004; Carvalho \& Ceccim, 2007; Ramos, 2009; González \& Almeida, 2010).

Objetivando-se a adequação dos egressos do sistema educacional às exigências de atuação do SUS, também se mostra pertinente a superação das dicotomias através da integração curricular e entre os campos de ensino e de prática. Nesse sentido, o diálogo entre as instituições de ensino e serviço em saúde; as práticas multiprofissionais e a coerência da formação às reais necessidades da população precisam pautar as modificações curriculares (Almeida \& Ferraz, 2008).

As DCN do curso de psicologia reconhecem o fenômeno psicológico como complexo e multideterminado e ainda asseguram uma formação crítica, generalista, plural e interdisciplinar. Além disso, definem a atenção à saúde psicológica e psicossocial com desenvolvimento de ações coletivas e individuais, não restringindo a psicologia ao campo da saúde mental e abrangendo a prevenção, promoção e reabilitação como campo de compreensão, análise e intervenção do profissional psicólogo. A relação entre a teoria e a prática também é destacada nas diretrizes, possibilitada preferencialmente pelo estágio curricular que insere o estudante no contexto de atuação profissional, consolidando as competências necessárias para o exercício da profissão (Resolução DCN, 2004).

Conforme diretrizes do Ministério da Educação, temos traçado o perfil profissional do psicólogo com diversas habilidades e competências constituintes da proposta de formação generalista, ainda que com ênfases curriculares. Contudo, temos uma profissão que transita entre os saberes da saúde e das ciências humanas e sociais e que também se situa entre as profissões liberais. Essa característica híbrida também se reflete no curso, que se constitui por cenários educativos pulverizados em diversas áreas, como a da psicologia organizacional e do trabalho, a psicologia do desenvolvimento, a psicologia educacional, a avaliação psicológica, as psicoterapias, dentre outras, além da saúde pública.

O projeto do curso, em vigor até 2009 , situa a demanda de serviços de saúde diante da crescente expectativa de vida da população e o acesso aos serviços de psicologia, apontando a conquista paulatina de inserção dos psicólogos nos serviços públicos em equipes de saúde, com ampliação das práticas tanto em 
saúde geral quanto na saúde mental, tanto na assistência quanto na gestão, no planejamento e na avaliação. É explicitada ainda, além da necessidade de desenvolver competências para a área, a compreensão sobre o lugar do profissional de psicologia na promoção de saúde (PUC, 2003).

De forma geral, ao descrever o perfil do psicólogo esperado, o projeto do curso de psicologia ressalta a atuação na promoção da saúde psíquica de sujeitos, de grupos, de organizações e de comunidades por meio de intervenções educativas, terapêuticas, psicossociais e também preventivas. Verifica-se que nas competências para tal perfil, também aparecem atuação na saúde na realização de atendimentos (individuais, de casal ou familiar) em consultórios ou outros estabelecimentos de saúde, além de planejamentos, atendimentos clínicos e projetos de intervenção e acompanhamento no setor da saúde, como ambulatórios, hospitais e outros (Batista, 2010).

A análise das ementas das disciplinas revela a presença do campo da saúde mental com conteúdos como psicopatologia, reforma psiquiátrica, políticas, rede de serviços substitutivos e práticas em saúde mental, além de estágios em serviços públicos e organizações não governamentais (ONG's).

$\mathrm{Na}$ interface com a saúde, outras ementas das áreas da Psicologia Social, do Trabalho e da Educação apresentam análises psicossociais e contribuições para a formação em saúde observadas nas disciplinas Psicologia e Políticas Públicas; Psicologia e Instituições de Saúder; Saúde Mental e Trabalho; e Psicologia e Sociedade Inclusiva. Além dessas, há outras disciplinas com debates da saúde pública que mesmo não explicitados em suas ementas, são desenvolvidos no curso, como Intervenção Psicossocial e Psicologia Social II.

O corpo docente do curso possui formação na área de saúde, com especializações, mestrado e doutorado, além de publicações atuais e experiências de atuação em CAPS/CERSAM (Centro de apoio Psicossocial/Centro de Referência em Saúde Mental), hospital psiquiátrico e hospital geral,

2 Esta disciplina aborda conteúdos sobre a política e os sistemas de saúde e a atuação do psicólogo em diversas instituições de saúde. No currículo implantado em 2010, a disciplina passa a ser chamada Psicologia e saúde, sofre pequenas alterações na ementa e deixa de ser ministrada no $10^{\circ}$ período para ser ministrada no 6 o período do curso.
Atenção Primária à Saúde (APS) e dependência química, principalmente. Áreas essas que também se configuram como estágios curriculares supervisionados, ofertados por esses professores.

No período analisado foram desenvolvidos cerca de 30 projetos de estágio específicos na saúde, intitulados: Intervenção psicossocial na saúde; Saúde Pública; Toxicomania; Políticas Públicas, Saúde Mental; Psicologia Hospitalar, Brinquedoteca no hospital; Crianças que chiam (ambulatório SUS); e Saúde mental e trabalho. Esses estágios são específicos da área da saúde, ainda que outros estágios, embora não tenham sido alvo do recorte dessa pesquisa, por vezes, apresentem interface com a saúde, ora pelos conteúdos abordados ora pelo local onde se realizam. Como exemplo, podemos citar os projetos de Intervenção com idosos, Estimulação cognitiva, Avaliação psicológica e Oficinas de grupo.

É importante esclarecer que o currículo do curso de psicologia proporciona um leque variado de cenários, inclusive de estágios que são planejados e ofertados conforme a proposta e estrutura do curso, em diálogo com: as necessidades sociais; a formação e o interesse por parte dos professores; a possibilidade dos campos de prática; e a demanda dos alunos. Nessa composição, as ofertas de estágios não são fixas nem permanentes e isso representa ora inovações, ora anacronismos.

Verificou-se uma diversidade de cenários de aprendizagem em saúde (Gráfico 1), em evidência os estágios curriculares, promoção e participação em eventos, apresentação de trabalhos em saúde e monografias, seguidos pelas publicações, e, por fim, projetos de pesquisa e de extensão. 0 desenvolvimento dos cenários apontados está concentrado em alguns professores, que interessados na área, diversificam suas práticas docentes no campo da saúde. Dois projetos de extensão foram desenvolvidos nesse período, um em serviços de saúde (APS) e outro em comunidades terapêuticas de tratamento de toxicômanos, além de cinco projetos de pesquisas em saúde. Das pesquisas, três delas são na área de APS e as demais em saúde mental e saúde e trabalho, todas com financiamento externo de agências de fomento ou promovidos em parceria com outra instituição pública. Os projetos de extensão estão articulados com estágios curriculares, através dos mesmos professores supervisores que realizam tanto os estágios quanto os projetos de extensão nas mesmas instituições e temáticas.

Psicol. Argum. 2014 jul./set., 32(78), 17-25 


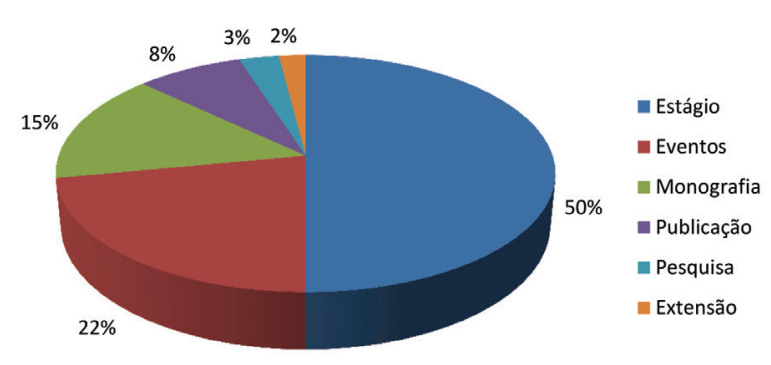

Gráfico 1 - Cenários de aprendizagem do curso de psicologia: tipo e frequência de cenários de aprendizagem de saúde

Os cenários de aprendizagem, em geral, estão distribuídos de forma regular entre três dos temas identificados no estudo, a saber: Saúde $e$ trabalho, Atenção Primária à Saúde (APS) e Saúde Mental, seguido da temática Hospitalar. A maioria dessas temáticas se refere à assistência em saúde, incluindo Drogadição e Ambulatório. Algumas outras temáticas como formação em saúde, saúde pública e saúde coletiva também aparecem no estudo, contudo, não foi identificada explicitamente nenhuma produção sobre gestão do trabalho, educação em saúde, controle social ou participação comunitária, temas recorrentes na literatura e apontados, também pela política de saúde, como necessários.

Dentre esses temas identificados, a temática Saúde e trabalho é bastante presente em todos os cenários de aprendizagem, com destaque nas monografias e eventos. Há também um número expressivo de produções de monografias sobre Saúde Mental. Nesses dois temas ainda se concentram as publicações, e o tema da Atenção Primária à Saúde (APS) apresenta o maior número de pesquisas. As pesquisas e as publicações na área de atenção primária refletem as apreensões e necessidades da política, dos gestores e de produções de conhecimentos sobre a promoção de saúde e o Programa de Saúde da Família.

No que se refere aos estágios (Gráfico 2), a área Hospitalar representa o maior número deles, entretanto essa temática é inexpressiva nos demais cenários de aprendizagem. Também com forte presença nos estágios nota-se os temas de Saúde mental e Atenção primária e, em seguida, Saúde e trabalho e Drogadição.

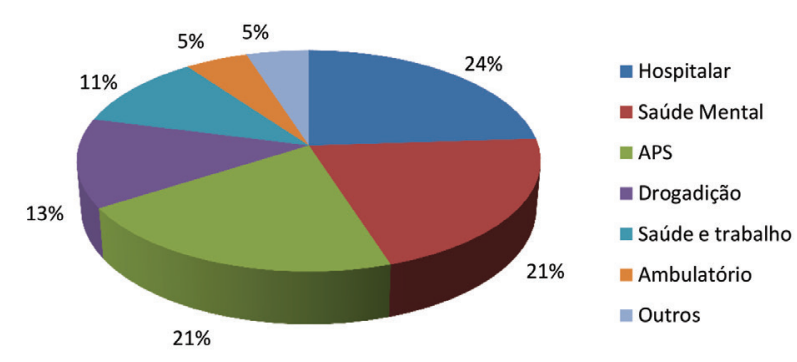

Gráfico 2 - Cenários de aprendizagem do curso de psicologia: frequência das temáticas em saúde nos estágios curriculares

Embora no período estudado tenhamos observado uma diversidade e um crescimento gradativo dos cenários de aprendizagem do curso voltados para a saúde pública, com presença de temáticas variadas, esses cenários estavam pouco articulados entre si ou mesmo com pouca continuidade em suas ações e campos de práticas. Contudo, devido ao caráter híbrido do curso de psicologia, há uma limitação no que tange à possibilidade de oferta de cenários e de número de professores específicos dessa área.

É possível observar que o início das parcerias da universidade com as Unidades Básicas de Saúde (UBS) é anterior ao período estudado, ${ }^{3}$ que foi se expandindo através da abertura de novos campos de estágio de psicologia.

No ano de 2008 houve um aumento dos cenários de aprendizagem em saúde, ano da implantação Pró-Saúde II na universidade com a participação dos professores do curso de psicologia. Nesse ano também aconteceu uma maior inserção de profissionais na Atenção Primária à saúde com contratações municipais de psicólogos no setor da saúde diante do estabelecimento do Núcleo de Apoio à Saúde da Família (NASF) pelo Ministério da Saúde (Portaria $\mathrm{n}^{\circ} 154,2008$ ).

Nota-se que as pesquisas e as publicações mais frequentes apresentadas no período investigado são sobre Atenção Primária, revelando sintonia com os incentivos e preocupações atuais no campo da saúde.

3 Esse quadro se altera com a ampliação e o fortalecimento das relações nos campos de estágios com a entrada do curso no Pró-Saúde em 2008 e com o novo currículo aprovado em 2009. 
A maioria dos estágios em saúde ocorre na rede pública e até a implantação do Pró-Saúde, o estabelecimento do vínculo entre a universidade e o serviço para tal atividade se caracterizava pela articulação dos próprios professores supervisores de estágios com a gerência das unidades básicas de saúde. Com isso, alguns cenários e práticas nesse período apresentam integração e continuidades frágeis. Esse quadro reafirma a proposta de reorientação da formação em saúde pautada na integração ensino-serviço, que, inclusive, já provocou aproximações e alguns acordos institucionais entre a universidade e a Secretaria Municipal de Saúde para o acompanhamento dos estágios em saúde na rede.

\section{Discutindo a formação em saúde}

Partindo dos preceitos da saúde coletiva, Carvalho e Ceccim (2007) identificam na formação universitária a necessidade de ampliar os cenários de aprendizagem incorporando a gestão, a atenção à saúde e o controle social; além de rever os conteúdos, as metodologias e a avaliação do ensino. E ainda verificar as orientações políticas e éticas dos projetos pedagógicos da graduação e de incentivar ações multiprofissionais em projetos de estágios, de pesquisa e de extensão.

Embora o estudo não tenha abordado todos estes pontos, alguns deles foram percebidos como a ação do corpo docente em constituir e diversificar os cenários educativos em saúde no curso de psicologia, principalmente associados ao serviço de saúde.

No intuito de uma formação generalista, a concentração de atividades de saúde identificada na pesquisa em alguns professores não nos parece problemática, desde que existam permanência e multiplicidade de cenários de aprendizagem em saúde, interação com os serviços, atuação multidisciplinar e integração entre ensino, pesquisa e extensão. Contudo, nos cenários universitários estudados há pouca presença de projetos multiprofissionais e pouca integração, ou ainda dispersão, entre ensino, pesquisa e extensão. Dessa forma, as críticas feitas aos cursos de graduação em saúde no que se refere às dificuldades ou poucas atividades multiprofissionais e ao frágil diálogo com outros saberes não nos parece diferente no referido curso de psicologia.

Conforme as DCN, a graduação de psicologia estudada oferece aprendizagem no campo da saúde pública com variados cenários que abarcam temáticas diferentes. De certo modo, há pontos de integração curricular retratados nas produções monográficas resultantes de estágios em saúde e na produção docente com publicações e apresentação de trabalhos, principalmente advindos de pesquisas (Batista, 2010).

A articulação entre o tripé da formação e a atuação multidisciplinar ainda representam um grande desafio não apenas para o curso de psicologia, mas para o ensino superior (Batista, Pereira, Matos, Andrade, Jorge, 2009). 0 próprio currículo universitário ainda hierarquiza essas três dimensões de aprendizagem e, além disso, a organização do conhecimento por disciplinas e por categorias profissionais dificulta ou mesmo inviabiliza práticas interdisciplinares e atuações em equipes multiprofissionais na universidade. Ainda que apresente projetos ou temas transversais, como modos instituintes de exceder a estrutura disciplinar como defende Albuquerque (2008), essa lógica permanece nos currículos.

O percurso de formação sustentado pelo currículo é organizado em tempos, espaços, corpo docente, conteúdos, metodologias, avaliações, projetos interdisciplinares, todos ancorados em concepções de sujeito e de aprendizagem refletidas nos cenários de aprendizagem. Esses ambientes de aprendizagem dentro do currículo assumem configurações múltiplas a partir da relação entre professores e alunos e seus contextos.

A complexa realidade curricular se constitui também por contradições, conflitos e relações de poder presentes nas relações sociais e na organização escolar. Desse modo, concordamos com Pacheco (2005) ao referir-se ao currículo como uma prática social, cultural e ideológica resultante de diversas forças. No caso dos cursos de saúde, podemos sinalizar as diferentes valorações sociais das profissões, a legitimação de conhecimentos específicos, as pressões do mercado e planos privados de assistência versus a consolidação do SUS, dentre outros pontos do contexto político-ideológico e econômico que repercutem nas relações na universidade e no currículo em prática.

Nessa direção, além de prática social, Silva (2003) afirma que o currículo é documento de identidade e relação de poder. Como instrumento e meio de constituir sujeitos, o currículo representa uma construção cultural e um percurso educativo organizado que ampara um plano de homem, de saúde, de sociedade em uma perspectiva que ultrapassa

Psicol. Argum. 2014 jul./set., 32(78), 17-25 
prescrições e se configura nas relações entre lugares, pessoas e instituições.

Acredita Ronzani (2007) que o currículo não pode mais ser visto como um conjunto neutro de conhecimentos e uma mera divisão entre aspectos técnicos e humanos envolvidos na formação. Sendo assim, a reforma curricular é movida por interesses e concepções em disputa, inclusive da própria proposta da política de saúde.

Compreendidos como prática social, os poderes em jogo não são apenas do Estado, da política pública e das ações estruturantes. Aliás, sozinhas elas não são suficientes para transformar os currículos, ainda que os programas e incentivos governamentais desempenhem papel fundamental de orientar as ações de mudança de modo extenso. Para tal transformação, devem também ser foco de análise e de mudança as relações institucionais e culturais ou ainda, segundo Ronzani (2007), as atitudes e as crenças que conformam a prática educativa e de saúde.

A implantação das diretrizes curriculares, a criação de programas para as graduações, como o Pró-Saúde e o PET-Saúde, e a abertura de postos de trabalho no SUS, como aquela provocada pela designação do NASF, alteraram o mapa curricular na universidade. No curso de psicologia, a mobilização de professores e de alunos para compreender o SUS e para participarem dos programas ministeriais, a aproximação entre o curso e os serviços de saúde com proposta de novos campos de estágios, o desenvolvimento de projetos de pesquisa e de extensão em saúde pública demonstraram ações e iniciativas de mudanças na formação em saúde.

\section{Considerações}

Estudar a formação em saúde oferecida nos cursos de graduação nos parece importante para superar alguns impasses ou mesmo críticas sobre o distanciamento da universidade em relação aos serviços de saúde e à sociedade, construir análises, compreensões e alternativas que contemplem a complexidade dessas relações. Além disso, o estudo permite sistematizar e avaliar aspectos da formação para futuramente identificar efeitos e impactos ocorridos com a participação nesse ou em outros programas.
A análise da configuração curricular e de seus cenários de aprendizagem do curso de psicologia atualiza/reafirma a reorientação da formação em saúde defendida pelas ações ministeriais, principalmente no que se refere à abordagem interdisciplinar, a atuação multiprofissional e a interação ensino-serviço.

$\mathrm{O}$ alcance das iniciativas e dos programas de mudança na formação em saúde requer mais estudos sistemáticos e longitudinais que analisem em sua complexidade, os processos e os produtos da reorientação sugerida. A formação e o envolvimento dos docentes e discentes, a inserção da clínica-escola como cenário de aprendizagem em saúde pública, os métodos e os conteúdos de ensino são aspectos a serem aprofundados em outras pesquisas, ampliando a compreensão da dimensão curricular na formação dos profissionais de saúde.

Os estudos sobre currículo sinalizam ainda a relevância desse documento como construção permanente de modos de aprender e ensinar, e também de questionar a própria política de saúde, as relações de trabalho e de profissionais no sistema de saúde, a abordagem e o vínculo com o usuário, dentre outros aspectos.

A formação forjada pelo currículo está inserida nos embates entre forças e grupos de interesses que se movimentam conforme as condições culturais, econômicas e políticas da sociedade. Assim, tornam-se também elementos da aprendizagem do profissional da saúde, além da gestão, financiamento e a participação social do SUS, as concepções de homem e de sociedade embutidas nesse projeto, considerando seu dinamismo, contradições e reinvenções.

\section{Referências}

Albuquerque, V. S. (20089) A integração Ensino-Serviço nos Processos de Mudança na Formação Superior dos Profissionais da Saúde. Revista Brasileira de Educação Médica, 32(3), 356-362. Recuperado em 05 de outubro, 2011, de http://www.scielo.br/scielo.php?pid=S0100-55022008000300010\&script=sci_arttext.

Almeida, L. P. von Gal de., \& Ferraz, C. A (2008). Políticas de formação de recursos humanos em saúde e enfermagem. Rev. bras. enferm, 61(1), 31-35. Recuperado em 04 de outubro, 2011, de http://www.scielo.br/scielo. php?pid=S0034-71672008000100005\&script=sci_arttext. 
Batista, C. B. (2010) A formação superior em saúde: currículo e cenários de aprendizagem no curso de psicologia da PUC Minas - unidade São Gabriel. Relatório de pesquisa. Belo Horizonte: FIP/PUC Minas.

Batista, C. B., Pereira, A. B., Matos, F. M. V. da S., Andrade, I. C., \& Jorge, K. B. F. (2009). Psicologia nas Unidades Básicas de Saúde: experiências de formação. In: Mayorga, C., Pereira, M. S., \& Rasera, E. F (orgs.). (pp. 251-265) Psicologia Social: sobre desigualdades e enfrentamentos. Curitiba: Juruá.

Becker, H. (1999) Observação Social e Estudos de Caso Sociais. Em Métodos de Pesquisa em Ciências Sociais. São Paulo: Editora Hucitec, pp. 117-133.

Borrione, R., \& Chaves, A. M (2004). Análise documental e contexto de desenvolvimento: estatutos de uma instituição de proteção à infância de Salvador, Bahia. Estudos de Psicologia, 21(2), 17-27.

Brasil. Ministério da Saúde [MS]. Ministério da Educação e Cultura [MEC]. (2009). Programa Nacional de Reorientação da Formação Profissional em Saúde Pró-Saúde: objetivos, implementação e desenvolvimento potencial. Ministério da Saúde, Ministério da Educação. Brasília: Ministério da Saúde.

Carvalho, Y. M. de, \& Ceccim, R. B (6 2007). Formação e educação em saúde: aprendizados com a saúde coletiva. In: Campos, G.W. et al. (Org.). Tratado de Saúde Coletiva (Vol. 1, $2^{\circ}$ Ed, pp. 137-170) São Paulo-Rio de Janeiro: Hucitec-Fiocruz

Ceccim, R. B., \& Feuerwerker, L. C. M (2004, setembro/ outubro). Mudança na graduação das profissões de saúde sob o eixo da integralidade. Cad. Saúde Pública, 20(5), páginas. Recuperado em 05 de outubro, 2011, de http://www.scielo.br/pdf/csp/v20n5/36.pdf.

Conselho Nacional de Educação. Câmara de Educação Superior. Resolução $n^{\circ}$ 8, de 7 de maio de 2004. Institui as Diretrizes Curriculares Nacionais para os cursos de graduação em Psicologia. Psicologia: Teoria e Pesquisa. Vol.20, n². Brasília, 2004, pp. 205-208. Recuperado em 10 de outubro, 2011, de http://www.scielo.br/pdf/ptp/ v20n2/a15v20n2.pdf.
González, A. D., \& Almeida, M. J. de (2010, Maio). Integralidade da saúde: norteando mudanças na graduação dos novos profissionais. Ciênc. saúde coleti$v a, 15(3)$ 757-762. Recuperado em 28 de setembro, 2011, de http://www.scielo.br/scielo.php?script=sci arttext\&pid=S1413-81232010000300018\&lng=en\&nr $\mathrm{m}=\mathrm{iso} \& \mathrm{t} \operatorname{lng}=\mathrm{pt}$.

Minayo, M. C. de S. (1994) O desafio do conhecimento: pesquisa qualitativa em saúde. São Paulo/Rio de Janeiro: Hucitec/Abrasco.

Pacheco, J. A. (2005) Currículo como construção cultura e social. Em Escritos curriculares. São Paulo: Cortez, pp. $57-78$

Portaria $n^{\circ} 154$ de 24 de janeiro de 2008. Cria os Núcleos de Apoio à Saúde da Família - NASF. Brasília, 2008. Recuperado em 10 de outubro, 2011, de http://www.brasilsus.com.br/legislacoes/gm/100677-154.html?q=

Pontifícia Universidade Católica de Minas Gerais (2003, Março). Projeto Educacional, Curso de Psicologia Unidades Coração Eucarístico, Betim e São Gabriel.

Pontifícia Universidade Católica de Minas Gerais (2008). Projeto Pró-saúde: Ações integradas de formação profissional em saúde, PUC Minas - SUS-BH.

Ramos, M. (2009, Junho) Educação pelo trabalho: possibilidades, limites e perspectivas da formação profissional. Saude soc.,18(supl. 2), 55-59.

Ronzani, T. M. (2007) A Reforma Curricular nos Cursos de Saúde: Qual o Papel das Crenças? Revista Brasileira de EducaçãoMédica31(1)38-43. Recuperadoem27desetembro, 2011, de http://www.scielo.br/scielo.php?script=sci arttext\&pid=S0100-55022007000100006\&lng=en\&nrm $=\mathrm{iso} \& \mathrm{t} \operatorname{lng}=\mathrm{pt}$.

Silva, T. T. da. (2003) Documentos de identidade: uma introdução às teorias do currículo. ( $2^{\mathrm{a}}$ ed. $5^{\mathrm{a}}$ reimpressão) Belo Horizonte: Autêntica.

Psicol. Argum. 2014 jul./set., 32(78), 17-25 